\title{
COVID-19 infection and cardiometabolic complications: short- and long-term treatment and management considerations
}

\author{
Lee Stoner ${ }^{1,2, *}$, Mark Faghy ${ }^{2,3,4}$, Ryan Conners ${ }^{2,5}$ \\ ${ }^{1}$ Department of Exercise and Sport Science, University of North Carolina at Chapel Hill, Chapel Hill, NC 27599, USA \\ ${ }^{2}$ Healthy Living for Pandemic Event Protection (HL-PIVOT) Network, Chicago, IL 60612, USA \\ ${ }^{3}$ Human Sciences Research Centre, University of Derby, DE22 1GB Derby, UK \\ ${ }^{4}$ Department of Physical Therapy, College of Applied Sciences, University of Illinois at Chicago, Chicago, IL 60607, USA \\ ${ }^{5}$ Department of Kinesiology, The University of Alabama in Huntsville, Huntsville, AL 35899, USA \\ *Correspondence: stonerl@email.unc.edu (Lee Stoner)
}

\section{DOI:10.31083/j.rcm2202031}

This is an open access article under the CC BY 4.0 license (https://creativecommons.org/licenses/by/4.0/).

Submitted: 8 May 2021 Revised: 8 May 2021 Accepted: 19 May 2021 Published: 30 June 2021

\section{Keywords}

Lifestyle; COVID-19; Healthcare; Cardiometabolic; Cardiovascular; Underserved populations; Public health; Global health

\section{Editorial introduction and purpose}

The ongoing and seemingly endless COVID-19 (SARSCoV-2) pandemic is not only a global health crisis, but it has also highlighted a number of pre-existing problems, including access to health care, greater severity of COVIDrelated complications for those with poor lifestyle behaviors of cardiometabolic diseases, and the added complexities underserved populations face with seeking and gaining access to essential healthcare services. Below we pose some of the many questions of interest to chronic disease management (summarized in Fig. 1), with an emphasis on cardiometabolic disease, during and following COVID-19. On behalf of HL-PIVOT (Healthy Living for Pandemic Event Protection) and Exercise is Medicine ${ }^{\circledR}$ (EIM) Underserved \& Community Health, this special issue aims to expand the discourse related to the short- and long-term impact of COVID19 on individual and public health. Below we pose some of the many topics of interest to chronic disease management, with an emphasis on cardiometabolic disease. during and following COVID-19. The intention of this section is to ignite interest in this special issue and spur continued discourse from authors across the biomedical spectrum.

\section{Topics of interest}

\subsection{Long-term cardiometabolic health complications}

Although the clinical manifestations of COVID-19 are dominated by respiratory symptoms, cardiometabolic physiology has not escaped the wrath of this virus. There are reports that cardiometabolic complications increase the likelihood of contracting COVID-19 as well as symptom severity. For example, a recent Cochrane review of 220 studies from across the globe, reported that hypertension (46\%), diabetes (22\%), and ischemic heart disease (11\%) are highly prevalent among those hospitalized for COVID-19 [1]. Additionally, the review reported that there has been a substantial incidence of a wide range of cardiovascular complications, including arrhythmias (9.3\%), heart failure (6.8\%) and thrombotic complications (7.4\%). It should be acknowledged that the Cochrane review focused on patients who were hospitalized. Whether or not cardiovascular complications occur in those infected with milder COVID-19 clearly warrants further attention. Additional questions that need to be addressed with respect to COVID-19-related cardiometabolic complications include: what is the underlying pathophysiology? Which populations are at heightened risk? What are the key risk factors? And what type of data is being collected to help address such questions?

\subsection{Lifestyle behaviors during and beyond COVID-19}

Wholesale restrictions were imposed by governments worldwide to slow the progression of the COVID-19 pandemic. Enforced lockdowns resulted in an immediate switch to remote and flexible working for billions worldwide, leading to immediate changes in lifestyle behaviors. One of the most highlighted issues is the effect of lockdowns on increased sedentary time and reduced physical activity. While recommendations for home-based exercise routines were promoted by leading health organizations [2, 3], reductions in population-level physical activity, health status and increased sedentary behavior has been observed [4]. A pertinent question and area of concern are whether activities and lifestyle behaviors enforced by the pandemic will return to pre-pandemic 'norms' or will the influence be sustained as part of a COVID-19 syndemic affecting population health and wellbeing? 


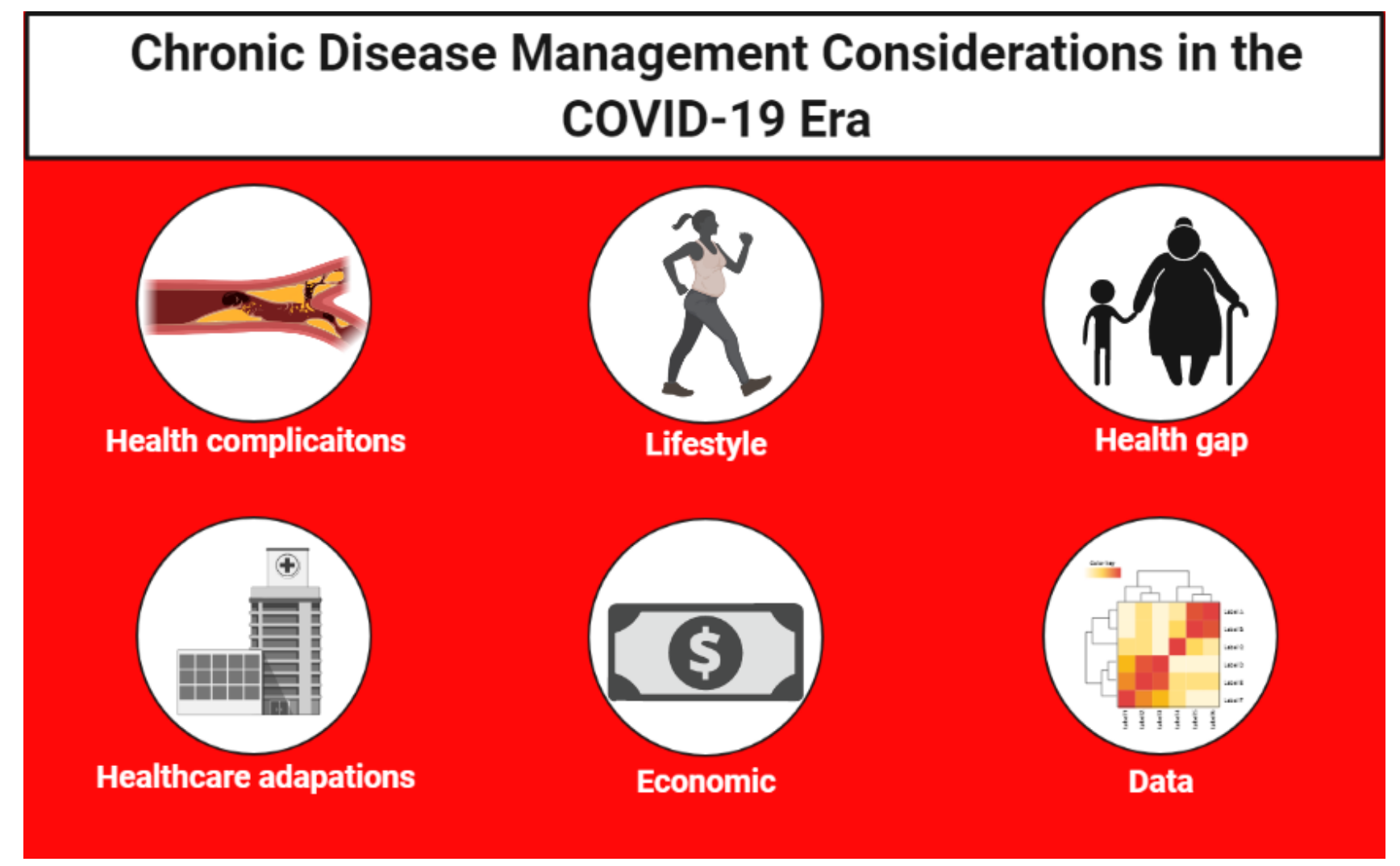

Fig. 1. Important considerations for managing chronic disease during and following the COVID-19 (SARS-CoV-2) pandemic include: (i) cardiometabolic health complications (e.g., what are the long-term impacts to vascular health?); (ii) lifestyle behaviors (e.g., will COVID-19 affect long-term lifestyle behavioral patterns?); (iii) the health gap (e.g., will COVID-19 widen the gap for underserved populations?); (iv) healthcare adaptations (e.g., what adaptations should be made to clinical practices?); (v) economic (e.g., will COVID-19 affect direct and indirect healthcare costs?); and (vi) the need for data (e.g., what data is being collected to help better understand the long-term cardiometabolic consequences of COVID-19 infection?).

\subsection{Health gap}

The broadening of health inequalities has been exposed by COVID-19, especially in those from underserved populations [5]. Existing inequalities, health epidemics and social determinants of health have rendered those from underserved populations at greater risk of COVID-19 infection, long-term morbidity, and mortality [6]. Sustained periods of transmission and the subsequent impact of prolonged recovery will undoubtedly pose long term risks to those from underserved communities, who undoubtedly will require prolonged access to health care services. Whilst efforts from international governments are prioritizing restoring the socio-economic factors affected by COVID-19, we believe efforts should also prioritize and address the widening health inequalities that could lead to an increase and widening of health inequalities.

\subsection{Healthcare adaptations}

The international response to COVID-19 has been drastic and immediate to prevent sustained progression and transmission of the virus. While international collaboration has led to the development of efficacious vaccination programs and gradual easing of international restrictions, the threat of COVID-19 and the lasting impact upon health status is everpresent. Healthcare providers and researchers are developing resources and support programs for a novel virus to cater for the broad challenges being experienced by patients following a COVID-19 infection [7], but the lasting impact has yet to be determined. Cardiovascular complications are part of a complex symptom profile that will require tailored support to improve and restore functional status to pre-COVID-19 levels [8]. The challenge and longitudinal impact will undoubtedly be a feature in clinical practice for the foreseeable future and we invite colleagues to consider the specific approaches in design and delivery of bespoke interventions. We also invite discussion pertaining to the long-term adaptations that will be needed in order to support the increasing and complex patient profile.

\subsection{National and global economies}

Prior to the COVID-19 pandemic, cardiovascular disease was the leading cause of death and the most costly in terms of health care expenditure [9]. As discussed in section 2.1, preexisting cardiometabolic complication not only place individual at risk for worse COVID-19 outcomes, the COVID-19 virus may lead to cardiovascular complications [1]. Moving forward, it remains unclear as to what the long-term impact will be in terms of COVID-19-related cardiovascular complications, and what the impact of such complications may 
be on direct healthcare costs, long-term rehabilitation costs, lost work days, and productivity losses? Additionally, what are the consequences to universal healthcare? And for countries such as the United States, which has a highly privatized healthcare system, what will be the ramifications for healthcare premiums [10]?

\subsection{The need for data}

The Covid-19 Effects on ARTErial StIffness and Vascular AgeiNg (CARTESIAN) study is a longitudinal international multicenter study investigating the long-term consequences of COVID-19 to vascular health [11]. A secondary objective of CARTESIAN is to evaluate whether the effects of COVID19 on the vascular system are moderate by psychosocial, comorbidities, and chronic treatment. Such data will be crucial in terms of identifying which factors influence the longterm vascular complications of CVOID-19, and for directing treatment strategies. Though additional data will be needed, including with respect to prevalence and risk factors among underserved population, identifying lifestyle strategies for mitigating the long-term cardiometabolic consequences of COVID-19, and identifying the best use of public health resources. Data is needed to address these and many additional foreseeable and unforeseeable questions. Our hope is that research teams will details the types of data they are using, preexisting questions being tackled with their data, and their policy for sharing data.

\subsection{Public health policy}

Across the globe, Goliath-esque challenges are being presented to public health agencies. These agencies will have a crucial role to play long following the ablation of COVID19 restrictions. Considering the widespread consequences of this [hopefully] once-in-a-lifetime pandemic, what types of policy should be implemented to ensure these agencies are best positioned to be proactive with foreseeable challenges and to react to unforeseen challenges? Which segments of the population should be primarily targeted? What forms of education are required to ensure the public is amenable to booster shots? Who should pay for booster shots?

\section{Conclusions}

Above we presented some of the many questions that need to be addressed to limit the short-and long-term cardiometabolic consequences of COVID-19. Our intention was not to be exhaustive, but rather to illuminate some of the many questions we, as a research community, need to address to be of service to the public. On behalf of HL-PIVOT and EIM we welcome stimulating and open discourse.

\section{Author contributions}

All authors conceptualized, drafted and approved this manuscript.

\section{Ethics approval and consent to participate \\ Not applicable.}

\section{Acknowledgment}

We would like to express our gratitude to all those who helped us during the writing of this manuscript.

\section{Funding}

This research received no external funding.

\section{Conflict of interest}

The authors declare no conflict of interest.

\section{References}

[1] Pellicori P, Doolub G, Wong CM, Lee KS, Mangion K, Ahmad M, et al. COVID-19 and its cardiovascular effects: a systematic review of prevalence studies. Cochrane Database of Systematic Reviews. 2021; 3: CD013879.

[2] World Health Organization. How to stay physically active during COVID-19 self-quarantine. 2021. Available at: https://www. euro.who.int/en/health-topics/health-emergencies/coronavir us-covid-19/publications-and-technical-guidance/noncommu nicable-diseases/stay-physically-active-during-self- quarantine (Accessed: 26 May 2021).

[3] Stanford K, Pomeroy A, Bates LC, Tamminga K, Chai T, Moore $\mathrm{JB}$, et al. Exercise is medicine ${ }^{\circledR}$ on campus during COVID-19: necessary adaptations and continuing importance. Translational Journal of the American College of Sports Medicine. 2020; 5: e000157.

[4] Faulkner J, O’Brien WJ, McGrane B, Wadsworth D, Batten J, Askew CD, et al. Physical activity, mental health and well-being of adults during initial COVID-19 containment strategies: a multicountry cross-sectional analysis. Journal of Science and Medicine in Sport. 2021; 24: 320-326.

[5] Bambra C, Riordan R, Ford J, Matthews F. The COVID-19 pandemic and health inequalities. Journal of Epidemiology and Community Health. 2020; 74: 964-968.

[6] Coronini-Cronberg S, John Maile E, Majeed A. Health inequalities: the hidden cost of COVID-19 in NHS hospital trusts? Journal of the Royal Society of Medicine. 2020; 113: 179-184.

[7] Venkatesan P. NICE guideline on long COVID. The Lancet Respiratory Medicine. 2021; 9: 129.

[8] Oronsky B, Larson C, Hammond TC, Oronsky A, Kesari S, Lybeck $\mathrm{M}$, et al. A review of persistent post-COVID syndrome (PPCS). Clinical Reviews in Allergy \& Immunology. 2021; 1-9.

[9] Virani SS, Alonso A, Aparicio HJ, Benjamin EJ, Bittencourt MS, Callaway CW, et al. Heart disease and stroke statistics-2021 update: a report from the american heart association. Circulation. 2021; 143: e254-e743.

[10] Zieff G, Kerr ZY, Moore JB, Stoner L. Universal healthcare in the united states of america: a healthy debate. Medicina. 2020; 56: 580.

[11] Bruno RM, Spronck B, Hametner B, Hughes A, Lacolley P, Mayer CC, et al. Covid-19 effects on arterial stiffness and vascular ageing: CARTESIAN study rationale and protocol. Artery Research. 2020; 27: 59-68. 